\title{
COMUNICAÇÃO
}

\section{OCORRÊNCIA DE Naupactus cervinus (Boheman) EM CAFEZAL NA REGIÃO DA ZONA DA MATA MINEIRA}

\author{
Occurrence of Naupactus cervinus (Boheman) in coffee plantation in the Region of Zona da Mata Mineira
}

\author{
Rodolfo Molinário de Souza ${ }^{1}$, Norivaldo dos Anjos², José Carlos Sorgato
}

\begin{abstract}
RESUMO
Naupactus cervinus é uma espécie altamente polífoga e já foi registrada causando danos a diversas culturas em vários Estados brasileiros. Objetivou-se, neste trabalho, registrar, pela primeira vez, a ocorrência de $N$. cervinus em cafeeiros na região da Zona da Mata Mineira, além de descrever os danos e a infestação numa plantação de café. Foi constatada uma infestação em rebrota de café, cultivar Catuaí, na região da Zona da Mata mineira. Ao se alimentarem, os adultos recortam os bordos das folhas novas deixando-as com aspecto de meia lua. Constatou-se uma média de $2,1 \pm 0,3$ insetos por planta e a grande maioria das plantas analisadas estava dentro da classe de plantas com menos de $25 \%$ de folhas atacadas.
\end{abstract}

Termos para indexação: Café, injúrias, besouro desfolhador, Curculionidae.

\begin{abstract}
Naupactus cervinus is a species highly polyphagous that has already been registered causing damages to many crops in several States of Brazil. This paper aims to register, for the first time, the occurrence of $N$. cervinus in coffee trees in the State of Minas Gerais, as well as to describe damages and infestation in a coffee plantation. An infestation was observed in a coffee sprout replantation, variety Catuaí, in the area of Zona da Mata of Minas Gerais. In feeding, adults of $N$. cervinus cut out the borders of tender leaves giving them an aspect of moon in first quarter. One verified an average of 2,1 $\pm 0,3$ insects for tree and the majority of analyzed plants was inside the class of plants with less than $25 \%$ of attacked leaves.
\end{abstract}

Index terms: Coffee, injuries, leaf eating beetle, Curculionidae.

(Recebido em 24 de julho de 2007 e aprovado em 4 de junho de 2008)

O gênero Naupactus Djean pertence à família Curculionidae, subfamília Entiminae. Dentro desta subfamília se destacam os coleópteros da tribo Naupactini, conhecidos como importantes pragas em diversas culturas. São insetos naturalmente distribuídos na região neotropical tendo alta diversidade em áreas tropicais e subtropicais da América do Sul (LANTERI \& MORRONE, 1995). Os principais estragos causados por essas espécies são provocados pelas larvas que vivem no solo e se alimentam das raízes (LANTERI et al., 2002), mas os prejuízos podem ser muito maiores por causa dos hábitos dos adultos de se alimentarem de folhas tenras, principalmente de plantas jovens (GUEDES et al., 2005).

A espécie Naupactus cervinus (Boheman), que, em trabalhos anteriores, já foi citada como Aramigus fulleri (Horn), Asynonychus godmanni (Crotch), Pantomorus olindae (Perkins), Naupactus simplex Pascoe, Pantomorus godmanni (Crotch), Strophomorphus canariensis Uyttenboogaart e Pantomorus cervinus (Boheman) (BEERS et al., 2003; BLOEM et al., 2002; LANTERI et al., 2002; SCATAGLINI et al., 2005) possui registros de ocorrência no Brasil, Argentina, Bolívia, Paraguai e Uruguai, tendo sido introduzida em países da América do Norte, África, Europa e Oceania (LANTERI et al., 2002). Considerada uma espécie altamente polífaga, no Brasil já foi registrada causando danos a diversas culturas nos estados de Minas Gerais, São Paulo, Paraná, Santa Catarina e Rio Grande do Sul (LANTERI et al., 2002). Segundo Lanteri et al. (1994), a espécie já foi observada se alimentando em várias plantas cultivadas, tais como fava (Vicia faba L.), batata-doce (Ipomoea batatas (L.)), pereira (Pyrus communis L.), damasco (Prunus armeniaca L.), erva-mate (Ilex

${ }^{1}$ Engenheiro Florestal, Mestre em Produção Vegetal - Laboratório de Manejo de Pragas Florestais - Departamento de Biologia Animal/DBA - Universidade Federal de Viçosa/UFV - Avenida Peter Henry Rolfs, s/n - Campus Universitário - 36570-000 - Viçosa, MG - molinariodesouza@yahoo.com.br ${ }^{2}$ Engenheiro Florestal, PhD em Entomologia, Professor - Laboratório de Manejo de Pragas Florestais - Departamento de Biologia Animal/DBA Universidade Federal de Viçosa/UFV - Avenida Peter Henry Rolfs, s/n - Campus Universitário - 36570-000 - Viçosa, MG - nanjos@ufv.br ${ }^{3}$ Graduando em Agronomia - Faculdade de Ciências Agrárias/FCA - Universidade Federal da Grande Dourados/UFGD - Rodovia Dourados à Itahum, Km 12 - Cx. P. 533 - 79804-970 - Dourados, MS - jc_sorgatto@hotmail.com 
paraguariensis St. Hil.), cana-de-açúcar (Saccharum officinarum L.), alfafa (Medicago sativa L.) e em Citrus sp. Costa \& Bogorni (1996) encontraram o inseto em duas espécies arbóreas na região de São Sepé (RS), a guaçatonga (Casearia sylvestris $\mathrm{Swartz}$ ) e a pitangueira (Eugenia uniflora L.). Além dessas plantas, uma infinidade de outras espécies vegetais já foram citadas como sendo atacadas por $N$. cervinus no Brasil e em outros países (BEERS et al., 2003; JUNQUEIRA 1952).

O inseto é um besouro de cor parda-clara quase uniforme, ligeiramente esbranquiçado, com uma listra longitudinal em cada lado dos élitros, mede entre oito e 10 milímetros e apresenta asas atrofiadas (BITANCOURT et al., 1933). Os adultos de $N$. cervinus se reproduzem por partenogênese (LANTERI \& NORMARK, 1995) e se alimentam durante a noite, permanecendo durante o dia escondido nas axilas das folhas ou entre essas (BITANCOURT et al., 1933). Ao se alimentarem, recortam os bordos das folhas deixando-as com o aspecto de meialua. A conseqüência das injúrias causadas nas folhas das plantas adultas é desconhecida, mas, em plantas jovens, tais injúrias podem resultar em uma maior perda de água e na diminuição da eficiência fotossintética, com conseqüente retardo em seu desenvolvimento (SYVERTSEN \& McCOY, 1985). O dano mais importante, entretanto, é aquele causado pelas larvas que consomem radicelas, raízes finas e a casca das raízes mais grossas. Segundo Junqueira (1952), quando a infestação de larvas é alta, além de amarelar as folhas e reduzir a taxa fotossintética, o imago serve ainda como vetor de moléstias fito-parasitárias, como a doença causada pelo fungo Cronartium ribicola Fischer.

Objetivou-se, neste trabalho, registrar, pela primeira vez, a ocorrência de $N$. cervinus em cafeeiros na região da Zona da Mata Mineira, além de descrever as injúrias e quantificar o nível de infestação causado pelos adultos numa plantação de café.

Adultos de $N$. cervinus foram coletados em março de 2007, em um cafezal localizado entre os municípios de Araponga e Canaã, região da Zona da Mata Mineira, com 10.000 pés de café da cultivar Catuaí, rebrota com quatro meses de idade e altura variando entre 35 e $45 \mathrm{~cm}$. O espaçamento utilizado foi de 4,0 X 1,5 m. Foram utilizadas para a identificação dos indivíduos as chaves dicotômicas propostas por Guedes et al. (2005) e Lanteri et al. (2002).

A área de rebrota está situada numa das partes mais altas do terreno ( $1300 \mathrm{~m}$ de altitude), sendo que dois lados da área são margeados por um plantio com cafeeiros em produção (plantas adultas) e os outros dois lados por vegetação rasteira nativa. Quarenta dias antes da vistoria foi feita, no local, uma aplicação de inseticida à base de deltametrina, mas houve uma reincidência dos besouros.

A avaliação das injúrias causadas por $N$. cervinus foi feita percorrendo-se três linhas que partiam de pontos diferentes dentro da área de rebrota, sempre da borda para o centro, perfazendo um total de 33 plantas por linha. A linha 1 de avaliação teve início a partir de um dos vértices localizado no ponto mais alto do terreno e margeado em ambos os lados por plantas adultas; a linha 2 partiu do ponto central de um dos lados margeados por cafeeiros em produção; e a linha 3 teve início a partir do ponto central de um dos lados margeados pela vegetação nativa. Durante essa avaliação foi obtida a incidência (quantidade de insetos por plantas) e avaliada a severidade (intensidade das injúrias nas plantas). A severidade foi obtida atribuindo-se a nota "0" quando nenhum sinal de ataque era observado; a nota " 1 " quando o cafeeiro apresentava menos de $25 \%$ de folhas atacadas, a nota " 3 " quando apresentava de 25 a $75 \%$ de folhas atacadas e a nota " 5 " quando mais de $75 \%$ das folhas estavam atacadas (Figura 1).

Registra-se, pela primeira vez, a ocorrência de Naupactus cervinus (Figura 2A) atacando cafezais em área de rebrota na região da Zona da Mata mineira. A espécie já foi constatada no Estado por Lanteri et al. (2002) em citros e por Fonseca (1946), na região de Poços de Caldas, em horta de tomate, ervilha e cará de cipó. A principal importância econômica de Naupactus cervinus está associada à citricultura, sendo que, em algumas regiões produtoras de citros dos estados de Minas Gerais e São Paulo, essa espécie possui o status de praga primária (GUEDES et al., 2005). Sua ocorrência em cafezais, no entanto, só havia sido registrada em cafeeiros do estado de São Paulo (FONSECA \& AUTUORI, 1932; JUNQUEIRA, 1952). Provavelmente, esse inseto já tenha causado danos em cafezais de outros estados em que é citado, mas tais informações não foram divulgadas, provavelmente por falta de conhecimento sobre a praga ou por não terem gerado perdas econômicas significativas.

As injúrias observadas nas folhas novas de café da área de rebrota são semelhantes àquelas citadas na literatura (FRANCE et al., 2002; JUNQUEIRA, 1952; STURMER et al., 2006). Os adultos fazem cortes em forma de meia-lua nas bordas das folhas, podendo consumir uma área foliar considerável (Figura 2B). Segundo France et al. (2002), $N$. cervinus têm hábitos gregários e se alimentam preferencialmente da margem das folhas, brotos, talos tenros e flores. Em folhas de citros, Guedes et al. (2007) registraram para $N$. cervinus um consumo foliar total de $15,0 \mathrm{~cm}^{2} \mathrm{de}$ folhagem. Segundo os autores, esse consumo pode representar pouco para a planta adulta, mas pode ser crítico para plantas jovens, por apresentarem área foliar reduzida, ou em condições de populações muito elevadas do inseto. 

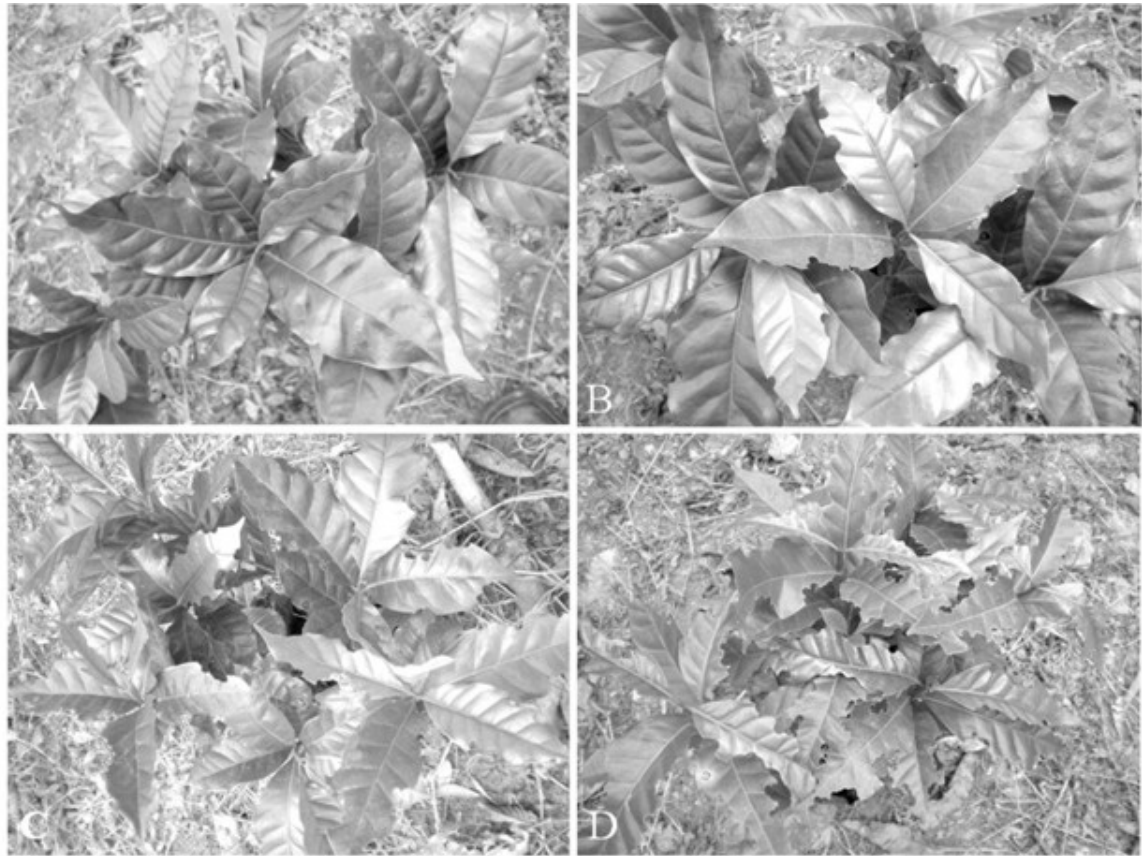

Figura 1 - Níveis de dano causado por Naupactus cervinus. Em (A) nenhum sinal de dano nas folhas, (B) menos de $25 \%$ de folhas danificadas, em (C) 25 a $75 \%$ e em (D) mais de $75 \%$ de folhas com sinal de ataque.
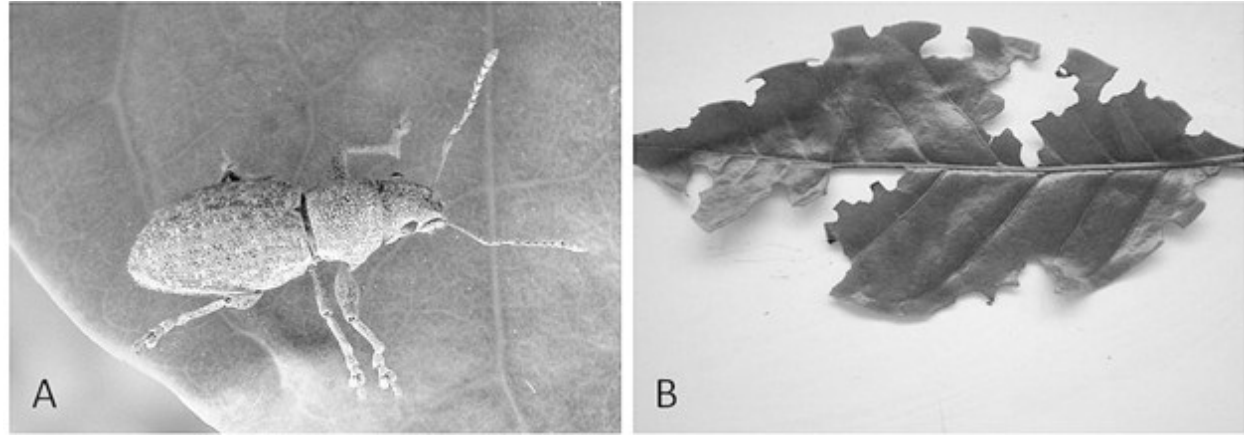

Figura 2 - (A) Adulto de Naupactus cervinus. (B) Injúrias causadas pelo adulto ao se alimentar das folhas de café.

A presença de $N$. cervinus em cafeeiros produtivos de áreas adjacentes também foi observada durante a vistoria, através da observação de folhas com injúrias semelhantes às descritas neste trabalho e pela presença esporádica de alguns adultos. Esses besouros, apesar de preferirem mudas e plantas jovens (GUEDES et al., 2005) também podem atacar plantas já desenvolvidas, iniciando o ataque, geralmente, pelas partes mais tenras e inferiores da planta (QUAYLE, 1941). A presença de plantas silvestres nas entrelinhas do café, com sintomas de ataque por $N$. cervinus, também foi verificada na vistoria, o que corrobora o fato de o inseto ser altamente polífago, tendo sido registrado alimentando-se de diversas espécies vegetais. Como os adultos necessitam se alimentar para produzir ovos, a utilização dessas plantas pode representar uma função significativa na biologia populacional, vindo a beneficiar a fecundidade e longevidade de $N$. cervinus (MAHER \& LOGAN, 2004).

Foi constatada uma incidência média de $2,1 \pm 0,3$ insetos por planta. Sendo que na linha três foram coletados em média mais insetos do que nas demais linhas (Tabela 1). 
Tabela 1 - Média da quantidade de adultos de Naupactus cervinus coletados e freqüência de folhas danificadas para as três linhas de avaliação.

\begin{tabular}{cccccc}
\hline \multirow{2}{*}{ Linha } & \multirow{2}{*}{$\begin{array}{c}\text { Média da quantidade de } \\
\text { besouros } \pm \text { erro padrão }(\mathrm{n}=33)\end{array}$} & \multicolumn{4}{c}{ Freqüência da intensidade de danos } \\
\cline { 3 - 6 } & $1,9 \pm 0,4$ & $0 \%$ & $<25 \%$ & 25 a $75 \%$ & $>75 \%$ \\
\hline L1 & $1,6 \pm 0,3$ & 0,27 & 0,61 & 0,12 & 0 \\
L2 & $2,6 \pm 0,6$ & 0,36 & 0,52 & 0,12 & 0 \\
L3 & $2,0 \pm 0,3$ & 0,24 & 0,48 & 0,27 & 0 \\
Média \pm erro padrão & $0,29 \pm 0,04$ & $0,54 \pm 0,04$ & $0,17 \pm 0,05$ & 0 \\
\hline
\end{tabular}

Em alguns casos, o nível de infestação foi bem alto, tendo sido observados até 12 indivíduos em uma única planta. Apenas um adulto de Artipus floridanus Horn (Coleoptera: Curculionidae: Entiminae) consome, em 72 horas, $4 \%$ da área foliar de uma folha de Citrus paradisi Macf. (SYVERTSEN \& McCoy, 1985). Conforme Bitancourt et al. (1933), os adultos de $N$. cervinus aparecem, geralmente, em número de 4 a 6 indivíduos por planta, contudo, se aparecerem em grandes quantidades, podem causar sérios danos às plantas novas. Para Sánchez-Soto et al. (2005) uma densidade de 3 a 5 indivíduos de $N$. curtus Boheman por planta é considerada baixa. Trabalhos que relacionem o consumo foliar com a densidade de insetos por planta devem ser estimulados para $N$. cervinus.

As plantas da linha 2 foram as que sofreram menor ataque por adultos de $N$. cervinus, com uma freqüência de 0,36 dentro da classe de $0 \%$ de folhas atacadas. Já as maiores freqüências de ataque em plantas com menos de $25 \%$ de intensidade de danos ocorreram dentro das linhas 1 e 3 e a freqüência de ataque de plantas dentro da classe de 25 a $75 \%$ de folhas danificadas foi maior na linha 3. Com isso, as linhas 1 e 3 foram as mais atacadas (Tabela 1). Não foram observadas em nenhuma linha plantas dentro da classe com mais de $75 \%$ de folhas danificadas. Entretanto, Junqueira (1952), ao registrar uma infestação de $N$. cervinus em 50.000 cafeeiros no estado de São Paulo, observou plantas totalmente atacadas. A ausência de plantas com severidade acima de $75 \%$ pode ser resultado do combate feito anteriormente com deltametrina.

Para conter a reinfestação foi utilizado um inseticida à base paration metílico. Segundo Guedes et al. (2002), ainda são desconhecidas informações sobre a biologia, ecologia, comportamento e distribuição da tribo Naupactini no Brasil, o que torna muitas vezes inviável o manejo racional e econômico desses insetos. Para os autores, a falta de tais informações resulta em um controle empírico e sem base em níveis de ação de inseticidas, com demanda de custos ambientais e econômicos elevados e com resultado pouco satisfatório, o que pode agravar ainda mais o problema.

Registrou-se pela primeira vez, para o estado de Minas Gerais as injúrias causadas por Naupactus cervinus a plantas de café da cultivar Catuaí, em uma área de rebrota localizada na região da Zona da Mata Mineira.

\section{AGRADECIMENTOS}

Ao proprietário do Sítio Itatiaia, Engenheiro Agrônomo Antônio Carlos Ribeiro, por disponibilizar a área para que fosse feita a vistoria e por fornecer informações sobre as condições locais. À Dra. Carolina Rocha da Silva e ao Biólogo Dinarte Gonçalves, pela ajuda na coleta de dados e informações.

\section{REFERÊNCIAS BIBLIOGRÁFICAS}

BEERS, E. H.; KLAUS, M. W.; GEBHARD, A.; COCKFIELD, S.; ZACK, R.; O'BRIEN, C. W. Weevils attacking fruit trees in Washington. In: ANNUAL WESTERN ORCHARD PEST \& DISEASE

MANAGEMENT CONFERENCE, 77., 2003, Washington,

DC. Proceedings... Washington, DC: Washington State

University, 2003. Disponível em: <http:// 'entomology.tfrec.wsu.edu/wopdmc/2003PDFs/ Rep $03 \% 20$ Biology $\% 20$ Beers.pdf 5 . Acesso em: 5 mar. 2003 .

BITANCOURT, A.; FONSECA, J. P.; AUTUORI, M.

Doenças, pragas e tratamentos. In: Manual de citricultura: II parte. São Paulo: Chácaras e Quintais, 1933. p. 140. 
BLOEM, S.; MIZELL, R. F.; O’BRIEN, C. W. Old traps for new weevils: new records for curculionids (Coleoptera: Curculionidae), brentids (Coleoptera: Brentidae) and anthribids (Coleoptera: Anthribidae) from Jefferson co., Florida. Florida Entomologist, Florida, v. 85, n. 4, p. 632 644, 2002.

COSTA, E. C.; BOGORNI, P. C. Insectos asociados al dosel del bosque secundario em Brasil: ColeopteraCurculionidae. Folia Entomológica Mexicana, Mexico, v. 98, p. 45-52, 1996.

FONSECA, J. P. Mais um inimigo da horta. Chácaras e Quintais, São Paulo, v. 73, n. 4, p. 432, abr. 1946.

FONSECA, J. P.; AUTUORI, M. Principais pragas do café no Estado de São Paulo. São Paulo: Escolas Profissionaes do Lyceu Coração de Jesus, 1932. 87 p.

FRANCE, A.; GERDING, M.; SANDOVAL, A. Patogenicidad de aislamientos chilenos de Beauveria bassiana en adultos de Asynonychus cervinus (Coleoptera: Curculionidae). Agricultura Técnica, Chile, v. 62, n. 4, p. 489-496, Oct. 2002.

GUEDES, J. C.; LANTERI, A. A.; PARRA, J. R. P. Chave de identificação, ocorrência e distribuição dos curculionídeos-das-raízes dos citros em São Paulo e Minas Gerais. Neotropical Entomology, Vacaria, v. 34, n. 4, p. 577-584, 2005.

GUEDES, J. C.; PARRA, J. R. P.; FIORIN, R. A. Aspectos biológicos da fase adulta dos curculionídeos-das-raízes dos citros. Ciência Rural, Santa Maria, v. 37, n. 2, p. 304307, 2007.

GUEDES, J. C.; PARRA, J. R. P.; YAMAMOTO, P. T. Levantamento da ocorrência de curculionídeos-dasraízes dos citros nos Estados de São Paulo e Minas Gerais. Laranja, São Paulo, v. 23, n. 2, p. 307-320, 2002.

JUNQUEIRA, G. M. Pantomorus godmani (Crotch): um depredador ocasional do cafeeiro. Solo, Piracicaba, v. 44, n. 2, p. 51-58, 1952.

LANTERI, A. A.; DÍAZ, N. B.; MORRONE, J. J. Identificación de las especies. In: LANTERI, A. A. (Ed.). Bases para el control integrado de los gorgojos de la alfalfa. La Plata: De la Campana, 1994. 119 p.
LANTERI, A. A.; GUEDES, J. C.; PARRA, J. R. P. Weevils injurious for roots of citrus in São Paulo State, Brazil. Neotropical Entomology, Vacaria, v. 31, n. 4, p. 561-569, Oct. 2002.

LANTERI, A. A.; MORRONE, J. J. Cladistics of the Naupactus leucoloma species group, Atrichonotus, and Eurymetopus (Coleoptera: Curculionidae). Revista de la Sociedad Entomológica Argentina, Mendoza, v. 54, p. 99-112, 1995.

LANTERI, A. A.; NORMARK, B. B. Parthenogenesis in the tribe Naupactini (Coleoptera: Curculionidae). Annals of the Entomological Society of America, Lanhan, v. 88, p. $722-731,1995$.

MAHER, B. J.; LOGAN, D. P. Comparison of host plant preferences, fecundity and longevity for dietreared and fiel-collected fuller's rose weevil. New Zeland Plant Protection Society, Hasting, v. 57, p. 183-190, 2004.

QUAYLE, H. J. Insects of citrus and other subtropical fruits. New York: Constock, 1941. 598 p.

SÁNCHEZ-SOTO, S.; GUEDES, J. C.; NAKANO, O. Ocorrência de Naupactus curtus Boheman (Coleoptera: Curculionidae) em três plantas de importância econômica no Brasil. Neotropical Entomology, Vacaria, v. 34, n. 4, p. 693, 2005.

SCATAGLINI, M. A.; LANTERI, A. A.; CONFALONIERI, V. A. Phylogeny of the Pantomorus-Naupactus complex base don morphological and molecular data (Coleoptera: Curculionidae). Cladistics, v. 21, p. 131-142, 2005.

STURMER, G. R.; GUEDES, J. V. C.; KARLEC, F.; SANTOS, J. C.; ARNEMANN, J. A.; WITTER, M. H. Ocorrência de Naupactus cervinus Boheman em citros em Santa Maria, RS. In: REUNIÃO ANUAL DA SBPC, 58., 2006, Florianópolis, SC. Anais... Florianópolis: SBPC, 2006. Disponível em: <http:// WWW.sbpcnet.org.br/livro/58ra/SENIOR/RESUMOSA resumo_3110.html I Acesso em: 1 maio 2006.

SYVERTSEN, J. P.; McCOY, C. W. Leaf feeding injury to citrus by root weevils adults: leaf area, photosynthesis, and water use efficency. Florida Entomologist, Florida, v. 68, n. 3, p. 384-393, 1985. 